\title{
NONUNIQUE CONTINUATION FOR UNIFORMLY PARABOLIC AND ELLIPTIC EQUATIONS IN SELFADJOINT DIVERGENCE FORM WITH HÖLDER CONTINUOUS COEFFICIENTS ${ }^{1}$
}

\author{
BY KEITH MILLER \\ Communicated by M. H. Protter, June 19, 1972
}

Consider the problem of backward uniqueness for the uniformly parabolic equation

$$
u_{t}=\sum_{i, j=1}^{n}\left(a_{i j}(x, t) u_{x_{j}}\right)_{x_{i}} \equiv \nabla \cdot \mathscr{A} \nabla u \quad \text { in } \Omega \times[0, \infty),
$$

$$
\mathscr{A} \nabla u \cdot v=0 \quad \text { on } \partial \Omega \times[0, \infty),
$$

and the problem of unique continuation (and uniqueness for the Cauchy problem) for the uniformly elliptic equation

$$
\sum_{i, j=1}^{n}\left(a_{i j}(x) u_{x_{j}}\right)_{x_{i}} \equiv \nabla \cdot \mathscr{A} \nabla u=0 \quad \text { in } \Omega,
$$

where $\Omega$ is a bounded domain in $R^{n}, v$ denotes the unit normal to $\partial \Omega$, and the symmetric matrix $\mathscr{A}$ has its eigenvalues in $\left[\alpha, \alpha^{-1}\right]$, with $\alpha>0$. We construct examples of nonuniqueness for (1) when $n=2$, and for (2) when $n=3$; in each case $\alpha$ may be arbitrarily close to 1 and the coefficients are also Hölder continuous.

Backward uniqueness for (1) with $\mathscr{C}^{1}$ coefficients was shown by LionsMalgrange [5]; probably the simplest proof is that of Agmon-Nirenberg [2] and Agmon [1] using the general method of logarithmic convexity. Carleman [4] long ago established unique continuation for (2) with $\mathscr{C}^{2}$ coefficients when $n=2$. For $n \geqq 3$, unique continuation for (2) with $\mathscr{C}^{2,1}$ coefficients was proved by Aronszajn [3], and more simply with $\mathscr{C}^{1}$ coefficients by Agmon [2]. See [1] and [6] for references to other results by Holmgren, Cordes, Hörmander, Landis, Lees and Protter, Bers and Nirenberg, and others.

An example of nonunique continuation was constructed by Plis [6] for a uniformly elliptic equation in the nondivergence form

AMS (MOS) subject classifications (1969). Primary 3537, 3542, 3562.

Key words and phrases. Parabolic backward uniqueness, elliptic unique continuation, Cauchy problem, heat conduction, ill-posed problems.

${ }^{1}$ Supported by a C.N.R. Visiting Professorship at Universitá di Firenze and by NSF grant GP 29369X. 


$$
\sum_{i, j=1}^{3} a_{i j}(x) u_{x_{i} x_{j}}+\sum_{i=1}^{3} b_{i}(x) u_{x_{i}}+c(x) u=0
$$

with Hölder continuous coefficients. Despite this example, the question of unique continuation for (2) has remained actively open. In the first place, it is well known that (2) and (3) often exhibit strikingly different behaviors when the coefficients become nonsmooth. In the second place, there is the strong physical interpretation of (1) and (2). These are the equations of time dependent (steady state) heat flow with variable nonisotropic conductivity matrix $\mathscr{A}$, with constant heat capacity, and with the conductivity in each direction bounded above and below. Condition (1)(b) implies that $\partial \Omega$ is totally insulated. The present results will be published in full elsewhere.

THEOREM 1. There exists an example of backward nonuniqueness on the halfspace $\Lambda=R^{2} \times[0, \infty)$ for a uniformly parabolic equation

$$
u_{t}=\left((1+A+a) u_{x}\right)_{x}+\left(b u_{y}\right)_{x}+\left(b u_{x}\right)_{y}+\left((1+C+c) u_{y}\right)_{y} \text { in } \Lambda \text {. }
$$

(i) The solution $u(x, y, t)$ is $\mathscr{C}^{\infty}$ on $\Lambda$, $\equiv 0$ for $t \geqq$ a certain positive $T$, but never $\equiv 0$ in any open subset of $R^{2} \times[0, T)$.

(ii) The coefficients $a(x, y, t), b(x, y, t), c(x, y, t)$ are $\mathscr{C}^{\infty}$ on $\Lambda$ and $\equiv 0$ for $t \geqq T$.

(iii) The coefficients $A(t), B(t)$ are Hölder continuous (here of order 1/6) on $[0, \infty), \mathscr{C}^{\infty}$ on $[0, T)$, and $\equiv 0$ for $t \geqq T$.

(iv) All functions $u, a, b, c$ are periodic $(2 \pi)$ in $x$ and $y$; $u$ is symmetric about $x=j \pi$ and $y=j \pi, j$ integer.

(v) Moreover, $u$ satisfies the "no flow" condition (1)(b) on $\partial \Omega \times[0, \infty)$, $\Omega=(0, \pi) \times(0, \pi)$, since both $b$ and the normal derivative $\partial u / \partial v$ are $=0$ there.

THEOREM 2. There exists an example of nonunique continuation on the halfspace $\Lambda=R^{2} \times[0, \infty)$ for a uniformly elliptic equation

$$
u_{t t}+\left((1+A+a) u_{x}\right)_{x}+\left(b u_{y}\right)_{x}+\left(b u_{x}\right)_{y}+\left((1+C+c) u_{y}\right)_{y}=0 \text { in } \Lambda .
$$

Conditions (i)-(v) on u, a, b, c, A, C hold exactly as stated in Theorem 1.

Outline of the parabolic example. Our construction proceeds with an $\infty$ of steps of successively shorter duration $T_{1}, T_{2}, \ldots$ whose sum $T$ is finite. Let us consider the $n$th step, in which the solution begins proportional to $\cos \lambda_{n} x \cdot \exp \left(-\lambda_{n}^{2} t\right)$, ends proportional to $\cos \lambda_{n+1} y \cdot \exp \left(-\lambda_{n+1}^{2} t\right)$, and at intermediate times is always a linear combination of $\cos \lambda_{n} x$ and $\cos \lambda_{n+1} y$. Here $\lambda_{1}, \lambda_{2}, \ldots$ is an increasing sequence of integers, to be chosen later. Each step will consist of three major phases (sandwiched 
between four "transition" phases needed for purposes of smoothness only). These seven phases have durations $\lambda_{n}^{-2}, \lambda_{n}^{-2}, \lambda_{n}^{-2}, s_{n} \lambda_{n}^{-2}, \lambda_{n}^{-2}, \lambda_{n}^{-2}, \lambda_{n}^{-2}$ respectively, where $s_{n}$ is a certain sequence tending to $\infty$, also to be chosen later. We define $\mu_{n}=\lambda_{n+1} / \lambda_{n}$ and $\varepsilon_{n}^{2} \equiv \exp \left(-\left(\mu_{n}^{2}-1\right) s_{n}\right)$ and point out that $\mu_{n}$ will be $\approx 1$ and $\varepsilon_{n} \approx 0$, especially for large $n$.

Let $\eta$ be a fixed $\mathscr{C}^{\infty}$ function on $[0,1]$ such that $\eta(t)$ is $\equiv 0$ near $t=0$, monotone on $[0,1]$, and $\equiv 1$ near $t=1$. We now proceed to list the duration, solution, and coefficients $1+A+a, b, 1+C+c$ of the three major phases. For convenience we start the time out anew with $t=0$ at the beginning of each phase and also employ the notation " ", "is proportional to".

The "transition 1" phase merely changes the coefficients smoothly from an initial $1,0,1$ to a final $1,0, \mu_{n}^{-2}$.

The "seed" phase has duration $\lambda_{n}^{-2}$, solution

$$
u \sim \cos \lambda_{n} x \cdot \exp \left(-\lambda_{n}^{2} t\right)+\eta\left(\lambda_{n}^{2} t\right) \varepsilon_{n} \cos \lambda_{n+1} y \cdot \exp \left(-\lambda_{n}^{2} t\right),
$$

and coefficients $1+a, b, \mu_{n}^{-2}$, where $a(x, y, t)$ and $b(x, y, t)$ will be described later. This phase serves to introduce a tiny $\cos \lambda_{n+1} y$ component into the solution.

The "transition 2" phase merely changes the decay rate of the first component smoothly from an $\exp \left(-\lambda_{n}^{2} t\right)$ rate to an $\exp \left(-\lambda_{n+1}^{2} t\right)$ rate.

The "distorted decay" phase has duration $\lambda_{n}^{-2} s_{n}$, solution

$$
u \sim \cos \lambda_{n} x \cdot \exp \left(-\lambda_{n+1}^{2} t\right)+\varepsilon_{n} \cos \lambda_{n+1} y \cdot \exp \left(-\lambda_{n}^{2} t\right),
$$

and coefficients $\mu_{n}^{2}, 0, \mu_{n}^{-2}$. This phase reverses the initial 1 to $\varepsilon_{n}$ ratio of the two components to a final $\varepsilon_{n}$ to 1 ratio.

The "transition 3" phase merely changes the decay rate of both components smoothly to the same $\exp \left(-\lambda_{n+1}^{2} t\right)$ rate.

The "removal" phase has duration $\lambda_{n}^{-2}$, solution

$$
u \sim \eta\left(\lambda_{n}^{2}(1-t)\right) \varepsilon_{n} \cos \lambda_{n} x \cdot \exp \left(-\lambda_{n+1}^{2} t\right)+\cos \lambda_{n+1} y \cdot \exp \left(-\lambda_{n+1}^{2} t\right),
$$

and coefficients $\mu_{n}^{2}, b, 1+c$, where the $b(x, y, t)$ and $c(x, y, t)$ will be described later. This phase removes a tiny component from the solution.

The "transition 4" phase merely changes the coefficients smoothly from $\mu_{n}^{2}, 0,1$ to $1,0,1$.

Derivation of the $a, b$. It is convenient to normalize the geometry, expanding the $x, y$, and $t$ scales by factors of $\lambda_{n}, \lambda_{n+1}$, and $\lambda_{n}^{2}$ respectively. That is, we consider $\tilde{u}$ defined by

$$
\tilde{u}(x, y, t) \equiv u\left(x / \lambda_{n}, y / \lambda_{n+1}, t / \lambda_{n}^{2}\right) \sim \cos x \cdot e^{-t}+\eta(t) \varepsilon_{n} \cos y \cdot e^{-t},
$$


which must be the solution of an equation with coefficients $1+\tilde{a}, \tilde{b}, 1$. Since $\cos x \cdot e^{-t}$ and $\cos y \cdot e^{-t}$ are already solutions of the equation with coefficients $1,0,1$, we are led to the following perturbation equation:

$$
(\tilde{a} \sin x)_{x}=-\eta \varepsilon_{n} \sin y \tilde{b}_{x}-\sin x \tilde{b}_{y}-\eta^{\prime} \varepsilon_{n} \cos y .
$$

By considering $\tilde{b}$ of the form $f(y) \cdot s(x, y)$ where $s$ is a certain solution of the first order PDE

$$
\eta \varepsilon_{n} \sin y \partial s / \partial x+\sin x \partial s / \partial y=0,
$$

one can construct a $\tilde{b}$ as desired such that the right-hand side of (7) has mean value zero across horizontal lines between $x=j \pi$ and $x=(j+1) \pi$. This $\tilde{b}$ inserted in (7) then determines an $\tilde{a}$ as desired. Moreover, each derivative of $\tilde{a}$ and $\tilde{b}$ is bounded by a constant times $\varepsilon_{n}$.

Construction of $b$ and $c$ for the "removal" phase is completely analogous.

Putting the pieces together. One now stacks the solutions and coefficients for the various phases and steps "end to end," after first multiplying the formulae $\left(u \sim \cos \lambda_{n} x \cdot \exp \left(-\lambda_{n}^{2} t\right)\right.$, etc.) in each phase by an appropriate magnitude constant in order to maintain continuity. With the proper choice of $\lambda_{n}$ and $s_{n}$, we can then make $T \equiv \sum T_{n} \equiv \sum\left(6+s_{n}\right) \lambda_{n}^{-2}$ finite, $u$ and the $a, b, c \rightarrow 0$ (in $\mathscr{C}^{\infty}$ fashion) and the $A(t), C(t) \rightarrow 0$ (in Hölder continuous fashion) as $t \rightarrow T$. It suffices for example to choose $\lambda_{n}=$ $(n+N)^{3}, s_{n}=(n+N)^{4}$, where the integer $N$ is taken sufficiently large to keep $\varepsilon_{n}, a, b, c, A, C$ small also during the initial steps. One then sets $u \equiv a \equiv b \equiv c \equiv A \equiv C \equiv 0$ for $t \geqq T$.

The elliptic example. The elliptic construction is extremely similar to the parabolic case. The solution in the $n$th step begins proportional to $\cos \lambda_{n} x \cdot \exp \left(-\lambda_{n} t\right)$ and ends proportional to $\cos \lambda_{n+1} y \cdot \exp \left(-\lambda_{n+1} t\right)$. The perturbation equation for $\tilde{a}$ and $\tilde{b}$ in the "seed" phase turns out to be in essentially the same form as (7) and the "transition 2" and "transition 3" phases require a bit more attention. We may choose $\lambda_{n}=(n+N)^{6}$ and $s_{n}=(n+N)^{4}$.

\section{REFERENCES}

1. S. Agmon, Unicité et convexité dans les problèmes différentiels, Séminaire de Mathématiques Supérieures, no. 13 (Eté, 1965), Les Presses de l'Université de Montréal, Montréal, Québec, 1966. MR 40 \#6025.

2. S. Agmon and L. Nirenberg, Properties of solutions of ordinary differential equations in Banach space, Comm. Pure Appl. Math. 16 (1963), 121-239. MR 27 \# 5142.

3. N. Aronszajn, A unique continuation theorem for solutions of elliptic partial differential equations or inequalities of second order, J. Math. Pures Appl. (9) 36 (1957), 235-249. MR 19, 1056. 
4. T. Carleman, Sur un problème d'unicité pour les systèmes d'équations aux dérivées partielles à deux variables indépendantes, Ark. Mat. Astr. Fys. 26B (1939), no. 17, 1-9. MR 1, 55 .

5. J. Lions and B. Malgrange, Sur l'unicité rétrograde dans les problèmes mixtes paraboliques, Math. Scand. 8 (1960), 277-286. MR 25 \# 4269.

6. A. Plis, On non-uniqueness in the Cauchy problem for an elliptic second order differential equation, Bull. Acad. Polon. Sci., Ser. Sci. Math. Astronom. Phys. 11 (1963), 95-100. MR 27 \#3920.

Department of Mathematics, University of California, Berkeley, California 94720 